\title{
Relative fluorine concentrations in radio frequency/electron cyclotron resonance hybrid glow discharges
}

\author{
M. L. Passow, a) J. T. P. Pender, and M. L. Brake \\ Department of Nuclear Engineering, The University of Michigan, Ann Arbor, Michigan 48109-2104 \\ K. T. Sung, Y. Liu, S. W. Pang, and M. E. Elta \\ Solid State Electronics Laboratory, Department of Electrical Engineering and Computer Science, \\ The University of Michigan, Ann Arbor, Michigan 48109-2122
}

\section{(Received 9 August 1991; accepted for publication 27 November 1991)}

\begin{abstract}
The relative concentration of atomic fluorine was measured in a radio frequency (rf) glow discharge and a modified electron cyclotron resonance microwave/rf hybrid discharge in $\mathrm{CF}_{4}$ using an actinometric technique. The dependence of fluorine concentration on if and microwave power, pressure, flow, and excitation source are presented. Anomalous behavior with rf power at constant microwave power was observed when using the Ar 750$\mathrm{nm}$ line as the actinometric species.
\end{abstract}

Combining a microwave source with a radio frequency (rf) system provides a means to independently control radical species production and ion bombardment energy. Conventional $\mathrm{rf}$ discharge reactors perform both tasks necessary for anisotropic etching, but in an interdependent fashion: (1) dissociation of feed gas into reactive radical constituents and (2) acceleration of ions onto the wafer surface. On the other hand, microwave electron cyclotron resonance (ECR) discharges are much more efficient at gas dissociation, but require elaborate magnetic confinement and/or biased ion extraction grids to produce directed ion streams. In a combined, or hybrid, system the microwave discharge region acts as the reactive species source and the rf discharge establishes the self-induced dc bias on the wafer chuck for ion acceleration. This arrangement is preferred over having biased ion extraction grids downstream of the ECR source which can contaminate the system.

To study the performance of the hybrid system, relative concentration trends of atomic $\mathrm{F}$ were measured in an rf glow discharge and a modified ECR/rf hybrid discharge in $\mathrm{CF}_{4}$ using the actinometric technique. This optical technique provides the relative concentration of reactive species. It was first introduced to study $\mathrm{CF}_{4}$ and $\mathrm{CF}_{4} / \mathrm{O}_{2}$ rf glow discharges ${ }^{1,2}$ and the specific application used here is outlined in Ref. 3. The dependence of $F$ concentration on rf and microwave power, pressure, flow, excitation source, and microwave cavity length was determined in both a pure rf and a hybrid system. The parameter space of interest was 3-20 sccm $\mathrm{CF}_{4}, 8.5-45 \mathrm{mTorr}, 20-400 \mathrm{~W}$ rf power, and $200-600 \mathrm{~W}$ microwave power. The $F$ trends in the hybrid system are compared with preliminary silicon etching results.

The experiments for this study were conducted on two systems. The first was a commercial $13.56-\mathrm{MHz}$ rf parallelplate radial flow reactive-ion etcher (RIE), a SEMI Group $1000 \mathrm{TP} / \mathrm{CC}$. The second system is similar to the first system, except the showerhead upper electrode was replaced with a Wavemat ECR, MPDR 325. The source

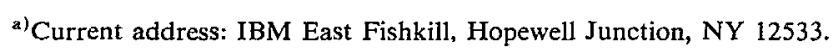

consisted of a 23 -cm-diam quartz disk with a gas ring at the base and a $37-\mathrm{cm}$-diam, adjustable length resonant cavity. Using a $2.45-\mathrm{GHz}$ microwave power supply, multimode excitation is expected for this cavity. ${ }^{4}$ Twelve rareearth magnets surrounded the discharge in an alternating pole configuration producing multipolar ECR zones. The etch gases were fed into the chamber through a distribution ring between the cavity base and the top of the chamber walls. The system was pumped with a Roots blower backed by a mechanical pump. This pumping package limited the processing pressure to be $>8 \mathrm{mT}$ Torr. Details of the experiment can be found in Refs. 5 and 6 .

The actinometric technique allows the relative concentration of ground-state atomic species to be determined under certain conditions independent of changes in the electron density and energy distribution function. This is achieved by introducing a small amount of an inert gas as an actinometer to which the reactive species emission is referenced. The specific requirements for the validity of this technique are as follows: (1) the emission from the two species must arise from deexcitation of higher-level states which are similar in energy; (2) the higher-energy states are created by direct electron impact excitation of ground-state atoms; and (3) the electron impact excitation cross sections are similar in shape and threshold energy. In this investigation, the actinometer was $\operatorname{Ar}$ (750 and 703 $\mathrm{nm})$ and the reactive species was $F(704 \mathrm{~nm})$. To minimize plasma perturbation from the addition of the actinometric gas, the argon flow rate was maintained at $5 \%$ of the $\mathrm{CF}_{4}$ flow. The emission signals were collected via an optical fiber. In the RIE system, emission was collected through a small window about $4 \mathrm{~cm}$ above the electrode. In the hybrid system emission was collected in two positions, through a quartz window on the side of the chamber $2 \mathrm{~cm}$ above the bottom electrode and through a viewing port on the microwave cavity which gave access to the quartz ECR containment disk.

Both the F and Ar emission, and hence the F concentration, changed as a function of cavity length and typically increased with decreasing cavity length. The variation of $\mathrm{F}$ concentration with residence time was examined by 


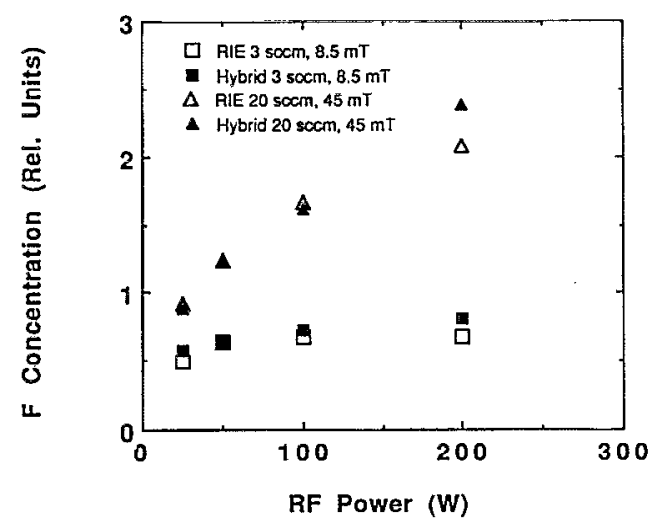

FIG. 1. F concentration as a function of $\mathrm{rf}$ power in both the RIE (open symbols) and the ECR/rf hybrid (closed symbol) systems for two cases, () $3 \mathrm{sccm}$ and $8.5 \mathrm{mT}$ and $(\Delta) 20 \mathrm{sccm}$ and $45 \mathrm{mT}$. Note there was no application of microwave power in the ECR/rf hybrid.

varying the pressure and flow rate. The $F$ concentration was expected to decrease with increasing flow or decreasing pressure since the residence time is less as has been observed by mass spectrometry measurements. ${ }^{7}$ As expected, the $F$ concentration in the $\mathrm{ECR} / \mathrm{rf}$ system decreased substantially for increased $\mathrm{CF}_{4}$ flow and increased with pressure in both the ECR/rf and RIE systems. In the RIE, at low pressure, the $F$ concentration remained approximately constant and at higher pressure the $\mathrm{F}$ concentration decreased with increasing flow. The residence time effect did not change the $F$ concentration greatly in the RIE due to the low overall $F$ production rate and the chamber loading effects which become important at low concentrations. ${ }^{3}$

In the RIE, the $F$ concentration increased with $\mathrm{rf}$ power as expected. To determine the effect of replacing the upper electrode with the ECR source, the F concentration was monitored as a function of $\mathrm{rf}$ power at the same conditions as the RIE. Even though the two reactors have very different geometries, the trends and magnitudes were approximately the same (see Fig. 1).

When microwave power was added to the hybrid system in addition to $50 \mathrm{~W}$ rf power, the $\mathrm{F}$ concentration increased substantially (see Fig. 2). Note the relative magnitude of the fluorine concentration can be an order of magnitude or more higher for the rf plus microwave power (Fig. 2) compared to the rf alone (Fig. 1). As the microwave power was increased, the $F$ concentration also increased as expected.

An anomalous behavior was observed when the rf power was varied in the hybrid system. With a constant microwave power of $200 \mathrm{~W}$, the atomic $\mathrm{F}$ concentration (i.e., the ratio of F $704 \mathrm{~nm}$ to Ar $750 \mathrm{~nm}$ ) decreased with increasing of power as shown in Fig. 3. This trend was also observed at higher microwave powers ( 250 and $400 \mathrm{~W}$ ), three $\mathrm{CF}_{4}$ flows $(3,10$, and $20 \mathrm{sccm}$ ), and $20-45 \mathrm{mTorr}$. Collecting the emission data directly from a viewport on the side of the microwave cavity also produced similar results. The same trend was observed with an $\mathrm{Al}$ electrode as well as a quartz-covered electrode. Note, however, that $\mathrm{Si}$ etch rates, which tend to follow the $\mathrm{F}$ concentration,

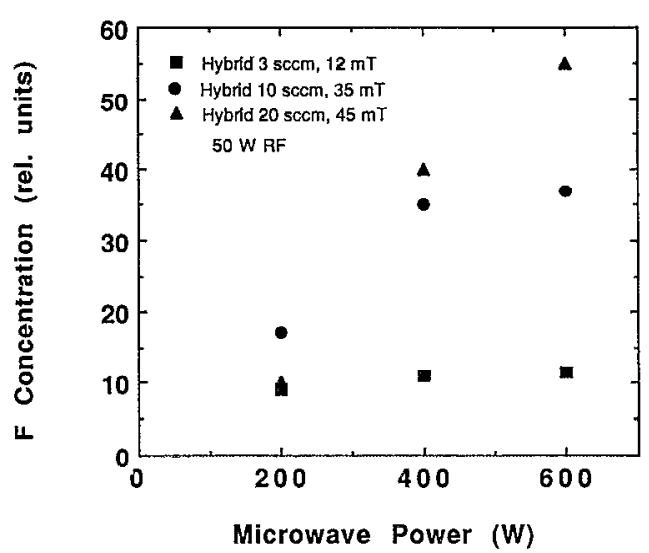

FIG. 2. F concentration as a function of microwave power in the ECR/rf system at (四) $3 \mathrm{sccm}$ and $12 \mathrm{mT}$, (†) $10 \mathrm{sccm}$ and $35 \mathrm{mT}$, and (A) 20 sccm and $45 \mathrm{mT}$ with $50 \mathrm{~W}$ rf power.

increased with increasing rf power under these operating conditions.

Increasing the $\mathrm{rf}$ power did increase the $\mathrm{F}$ emission intensity as well as the Ar emission intensity. 'The ratio, however, decreased due to the larger rate of increase of the argon emission intensity relative to the $F$ emission intensity. This effect may be due to the different excitation threshold energies of the lines. The F 704-nm line has a threshold energy of $14.79 \mathrm{eV}$ compared to $13.52 \mathrm{eV}$ for the Ar 750-nm line. If the electron energies increase with increasing if power, an enhancement in the population of the species with lower excitation thresholds can occur, depending upon the shape of the electron energy distribution function and the separation of the threshold energies.

Langmuir probe measurements ${ }^{8}$ indicate that the electron energy distribution function may be approximately Maxwellian in some ECR systems which may or may not apply here. We were not able to verify this assumption since a Langmuir probe was not available for this experiment, however, the electronic temperature of argon, which

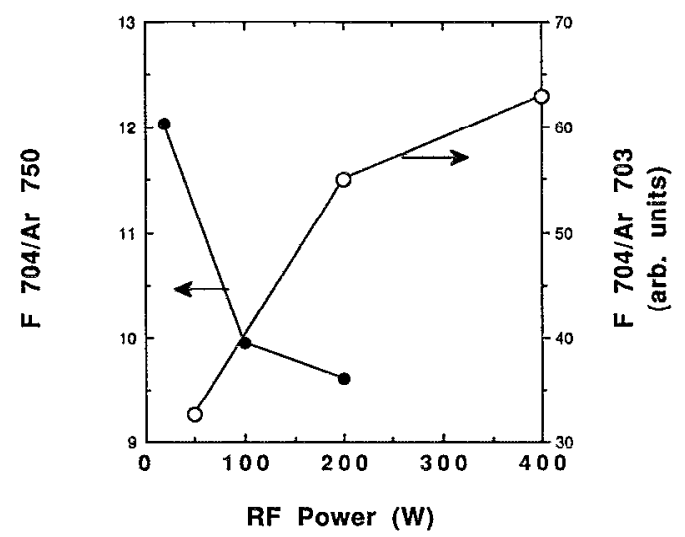

FIG. 3. F concentration as a function of if power in the ECR/ $\mathrm{I}$ [ system. The left-hand axis is the relative concentration obtained from $F 704 \mathrm{~nm} /$ Ar $750 \mathrm{~nm}$ and the right-hand axis is the relative concentration obtained from $\mathrm{F} 704 \mathrm{~nm} / \mathrm{Ar} 703 \mathrm{~nm}$ for $20 \mathrm{sccm}$ of $\mathrm{CF}_{4}, 45 \mathrm{mT}$, and $200 \mathrm{~W}$ of microwave power. 
assumes a Maxwellian distribution, could be estimated from atomic Boltzmann plots ${ }^{9}$ of the intensities of Ar lines $(706.7,750.4$, and $751.5 \mathrm{~nm})$. The electronic temperature of Ar was found to increase from 7800 to $9200 \mathrm{~K}$ as the rf power was increased from 20 to $200 \mathrm{~W}$ at $20 \mathrm{sccm} \mathrm{CF}_{4}$ and $45 \mathrm{~m}$ Torr. This may indicate an increase in the average electron energy which increases the excitation of the Ar 750-nm line (at $13.52 \mathrm{eV}$ ) preferentially over the higherenergy F 704-nm line (at $14.79 \mathrm{eV}$ ).

The Ar $703-\mathrm{nm}$ line at $14.88 \mathrm{eV}$ has a similar excitation threshold to the F 704-nm line but was very difficult to use because of its low intensity and interference from the $\mathrm{CF}_{x}$ continuum emission and the nearby, intense $\mathrm{F} 704-\mathrm{nm}$ line. However, for cases where the signal-to-noise was acceptable and when the continuum emission was subtracted from the Ar 703-nm line, the resulting $\mathrm{F}$ concentration trends increased as expected with increasing of power, as shown in Fig. 3.

In the hybrid $\mathrm{ECR} / \mathrm{rf}$ system, the actinometric results showed an increase in the $\mathrm{F}$ concentration as the microwave power and pressure were increased, which corresponds to an increase in Si etch rates. The Si etch rate also increased with rf power. The etch profiles indicated significant chemical etching as they were somewhat isotropic. Compared to etching in the RIE, higher Si etch rates were obtained in the hybrid ECR/rf system, which is in agreement with the actinometry results.

As described above, the ECR/rf system operates well in the traditional parameter range of the RIE. The hybrid system offers more flexibility and controllability than the RIE because it has a larger dynamic range of operating parameters and because it has the ability to independently adjust the reactive component using the ECR source and ion bombardment component using rf biasing. More work needs to be done, however, to assess the advantages for selectivity, damage, profile control, and uniformity.

This work is sponsored by the Semiconductor $\mathrm{Re}$ search Corp. (90-MC7-085). One of us (M.L.P.) wishes to acknowledge the support of an SRC Fellowship. We also gratefully acknowledge the technical assistance of Jeff Fournier. We would also like to thank Wavemat, Inc. for the usage of the ECR source and M. Dahimene for technical advice.

${ }^{1}$ J. W. Coburn and M. Chen, J. Appl. Phys. 51, 3134 (1980).

${ }^{2}$ R. d'Agostino, F. Cramarossa, S. DeBenedictis, and G. Ferraro, J. Appl. Phys. 52, 1259 (1981).

${ }^{3}$ T. J. Cotler, M. L. Passow, J. P. Fournier, M. L. Brake, and M. E. Elta, J. Appl. Phys. 69, 2885 (1991).

${ }^{4}$ J. Asmussen, J. Hopwood, and F. C. Sze, Rev. Sci. Instrum. 61, 250 (1990).

${ }^{5}$ M. L. Passow, Ph.D. thesis, University of Michigan, 1991.

${ }^{\circ} \mathrm{S}$. W. Pang, Y. Liu, and S. T. Sung, J. Vac. Sci. Technol. B 9, 3530 (1991).

${ }^{7}$ G. Smolinshky and D. L. Flamm, J. Appl. Phys. 50, 4982 (1979).

${ }^{8}$ Y. H. Lee, J. E. Heidenreich III. and G. Fortuno, J. Vac. Sci. Technol. A 7, 903 (1989).

${ }^{\prime}$ R. H. Tourin, Spectroscopic Gas Temperature Measurements (Elsevier, New York, 1966). 